\title{
Analysis on Nonlinear Stress-Growth Data for Shear Flow of Starch Material with Shear Process
}

\author{
Jinghu Yu, Dejun Ma, and Hui Lu \\ Jiangnan University, Lihu Road No. 1800, Wuxi, Jiangsu, China \\ Correspondence should be addressed to Jinghu Yu; jhyu@jiangnan.edu.cn
}

Received 3 May 2013; Accepted 29 May 2013

Academic Editor: Jun Wang

Copyright (C) 2013 Jinghu Yu et al. This is an open access article distributed under the Creative Commons Attribution License, which permits unrestricted use, distribution, and reproduction in any medium, provided the original work is properly cited.

The material function of liquid materials for packaging plays an important role in analysis of its mechanical behavior. The mechanical behavior of material affects the packaging process in many aspects, such as selection of packaging materials and preparation of packaging method. Therefore, research on the material function of the liquid material is very helpful to guide the packaging process and look into how the packaging quality and efficiency are affected by the mechanical properties of material. This paper established the material function for the starch solution under shear process. With the relaxation test of the starch solution specimens, the $G(t)$ function and dumping function were established and verified. Based on the memory function of starch solution, the material function of starch solution was constructed and approved to be efficiently predict the mechanical behavior during the shear process. Therefore, such material function can be used to guide the operation on the shear flow.

\section{Introduction}

Starches have been used for many years in the food industry, and their rheological properties decide different applications in food products $[1,2]$. For this reason, their rheological behavior has deserved increasing attention. The nonlinear behavior of starch solution plays an important role in many packaging processes, and especially it affects the packaging material transmission speed and high weight measurement precision $[3,4]$. Analysis of the nonlinear viscoelastic behavior of starch solution is of great importance for their rheological characterization.

The material function is the basis of the analysis on the starch solution. In order to establish its material function, the constitutive equation must be constructed according to the most suitable experiment [5]. It is often found that the Lodge model can successfully describe the behavior of rubber-like liquid under shear flow or elongational flow $[6,7]$. The Lodge model can be expressed as follows:

$$
\sigma(t)=-\int_{-\infty}^{t} M\left(t-t^{\prime}, \gamma\right) * \varepsilon\left(t^{\prime}\right) d t^{\prime}
$$

where $\sigma$ is the stress at the current time $t, M\left(t-t^{\prime}, \gamma\right)$ is the memory function specific for the material, and it can be established by suitable experiment. $\varepsilon\left(t^{\prime}\right)$ is the strain function dependent on the time $t$, and it can be defined in the specific process.

Recently, the memory function is often expressed as a product of the memory function for the linear behavior $G(t)$ and a so-called damping function $h(\gamma)$ [8-10]. Yu et al. [11] established and validated a rheological model that can be used to characterize viscoelastic properties of food gels during compression under small and large deformation [11]. The aim of this paper is to give experimental justification for linear behavior $G(t)$ and the damping function $h(\gamma)$. Taking the starch solution as sample, its material function was established based on the linear shear modulus $G(t)$ and damping function $h(\gamma)$. Through comparison of the experimental data and the calculated shear stress by the material function, the material function of starch solution was approved to be successfully describing the behavior of the starch solution under shear flow.

\section{Experimental Materials and Methods}

The type of modified starch is ULTRA-TEX 1 corn starch from National Starch Food Innovation. This kind of starch 


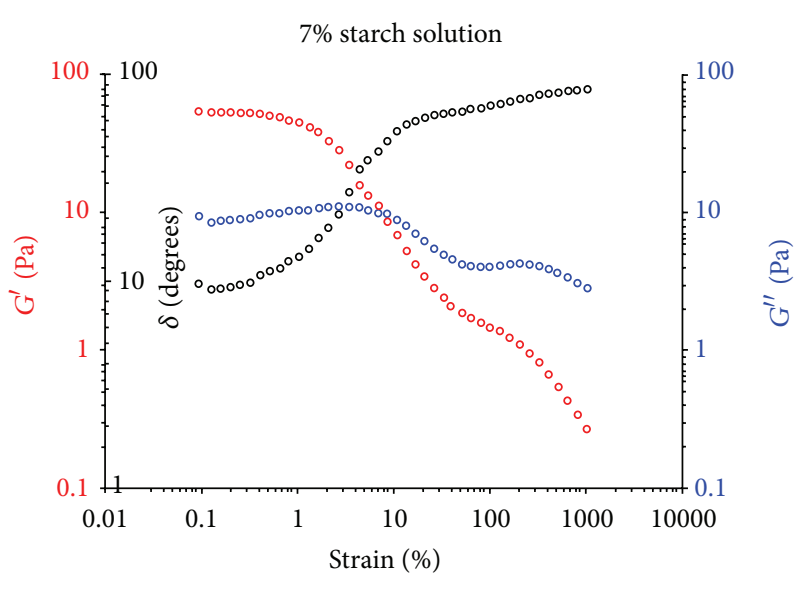

○० Strain sweep from 0.0001 to 10 strain sweep step

FIGURE 1: Strain sweep test from 0.0001 to 10 at the frequency 1.

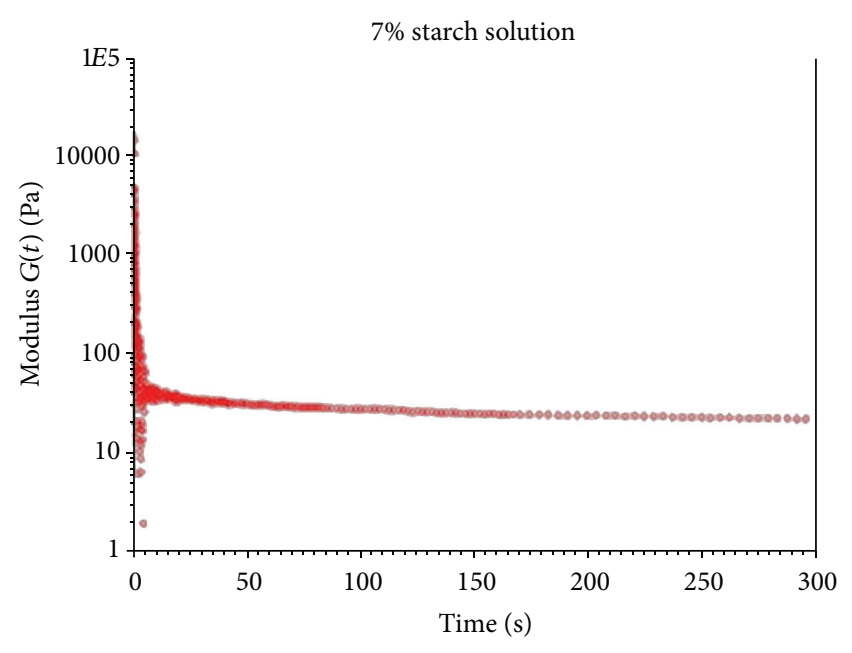

○ Strain is $0.3 \%$, stress relaxation test

Figure 2: Relaxation test within linear range of strain.

and distilled water were used to make different concentration starch solution. The whole experiment was performed on the Rheometer AR-2000 from TA instruments Ltd. The cone head was installed, and its angle is 2.8 degree. The constant test temperature is $25^{\circ} \mathrm{C}$.

High shear rate and long time shear test were performed to find if the shear process affects the strain relaxation test. Shear rate influence on the strain can be found. Therefore, the memory function can be established by the many different strain relaxation tests.

\section{Memory Function for the Nonlinear Viscoelastic Behavior}

The memory function can characterize the mechanical property of the specific material [12]. According to Wagner $[9,10]$, for simple shear flow the generalized memory function is expressed by

$$
M\left(t-t^{\prime}, \gamma_{t, t^{\prime}}\right)=\dot{M}\left(t-t^{\prime}\right) h\left(\gamma_{t, t^{\prime}}\right),
$$

where $\dot{M}\left(t-t^{\prime}\right)$ is the linear modulus of material under small deformation. It describes the linear relationship between the strain and stress of material. The nonlinearity of the rheological behavior is only characterized by the dumping function $h\left(\gamma_{t, t^{\prime}}\right)$.

For simple shear flow, the well known relations for the shear stress $\sigma(t)$ can be expressed by

$$
\sigma(t)=\int_{0}^{\infty} M\left(t-t^{\prime}\right) \gamma_{t, t^{\prime}} d t^{\prime} .
$$

In order to establish the shear modulus function and the damping function, the linear range of the relationship of the stress and strain must be found with strain sweep test. Figure 1 shows that the storage modulus $G^{\prime}$, the lost modulus $G^{\prime \prime}$, and different degree delta are almost constant when the strain is less than 0.01. It indicates that the relationship of stress and strain of starch solution is linear during the step in shear strain from 0 to 0.01 . Therefore, in the linear viscoelastic range the shear relaxation modulus $G(t)$ can be given by

$$
G(t) \approx G(t, \gamma)=\frac{\sigma(t)}{\gamma} .
$$

According to the strain sweep test result, the suitable strain relaxation test was carried out. The measurement data of relaxation test was shown in Figure 2. The strain value of relaxation test is $0.3 \%$, and it belongs to the linear viscoelastic range of $7 \%$ concentration starch solution.

The recently published paper that approved the linear modulus function can be expressed by $[13,14]$

$$
G(t)=a t^{-n} \text {. }
$$

Take the experiment data (Figure 2) to fit the function (5) with Origin 8.0, and the fitted linear modulus function can be expressed by

$$
G(t)=64.69095 t^{-0.19167} .
$$

The linear modulus function $G(t)$ characterizes the linear property of material during the small deformation. With certain deformation, the relationship of stress and strain is linear, and the shear modulus $G(t)$ is only dependent on time $t$. With the strain increasing, the relationship of stress and strain is nonlinear, and shear modulus $G(t, \gamma)$ is dependent on time and strain. The shear modulus function $G(t, \gamma)$ can be expressed by $[15,16]$

$$
G(t, \gamma)=G(t) h(\gamma),
$$

where $h(\gamma)$ is a damping function, and it is a function of strain $\gamma$. The damping function was added into the shear modulus to characterize the nonlinear property of material. It describes the relationship of the shear modulus and the strain. 
TABle 1: The value of strain at $t=50 \mathrm{~s}$ in the different strain relaxation test.

\begin{tabular}{lccccccc}
\hline Strain & $0.3 \%$ & $1 \%$ & $4 \%$ & $20 \%$ & $100 \%$ & $300 \%$ & $600 \%$ \\
\hline Strain $\gamma$ & 30.82 & 24.18 & 11.99 & 3.088 & 0.5657 & 0.1914 & 0.078 \\
\hline
\end{tabular}

TABLE 2: The value of dumping function at different strain.

\begin{tabular}{lccccccc}
\hline Strain & 0 & 0.007 & 0.037 & 0.197 & 1 & 3 & 6 \\
\hline$h(\gamma)$ & 1 & 0.7845 & 0.389 & 0.1 & 0.018 & 0.006 & 0.0025 \\
\hline
\end{tabular}

Derived from (7), $h(\gamma)$ can be expressed by

$$
h(\gamma)=\frac{G(t, \gamma)}{G(t)} .
$$

Many measurements of the relaxation modulus were performed in a nonlinear shear strain range of $\gamma=4 \%$ to $10 \%$ (Figure 3), where the starch solution behaves strongly nonlinear.

According to the published data of damping function $[17,18]$, the following equation can be selected as the starch solution's damping function:

$$
h(\gamma)=e^{-n \gamma} .
$$
follows:

Therefore, the relaxation modulus can be expressed as

$$
G(t, \gamma)=G(t) e^{-n_{1} \gamma}=a t^{-n_{2}} * e^{-n_{1} \gamma} .
$$

The measurements data was shown in Tables 1 and 2.

The dumping function can be fitted by the measurements data. The fitted result was shown as follow:

$$
h(\gamma)=e^{-26.38 \gamma} .
$$

Therefore, the shear modulus can be expressed as follow:

$$
G(t, \gamma)=64.69095 t^{-0.19167} * e^{-26.38 \gamma} .
$$

\section{Calculation of Nonlinear Material Functions}

The material function of starch solution can be established based on the Lodge model, and the stress under small deformation can be expressed by

$$
\sigma(t)=\int_{-\infty}^{t} G\left(t-t^{\prime}, \varepsilon\left(t^{\prime}\right)\right) * \dot{\varepsilon}\left(t^{\prime}\right) d t^{\prime} .
$$

Take (12) into (13), and the material function of starch solution can be expressed by

$$
\sigma(t)=\int_{-\infty}^{t} 64.69095 t^{\prime^{-0.19167}} * e^{-26.38 \gamma} * \dot{\varepsilon}\left(t^{\prime}\right) d t^{\prime},
$$

where $\gamma$ is the current strain. The shear history can be described by the following:

$$
\gamma_{t, t^{\prime}}= \begin{cases}\dot{\gamma}_{0}\left(t-t^{\prime}\right) & \text { for } t-t^{\prime}<t \\ \dot{\gamma}_{0} t & \text { for } t-t^{\prime} \geq t .\end{cases}
$$

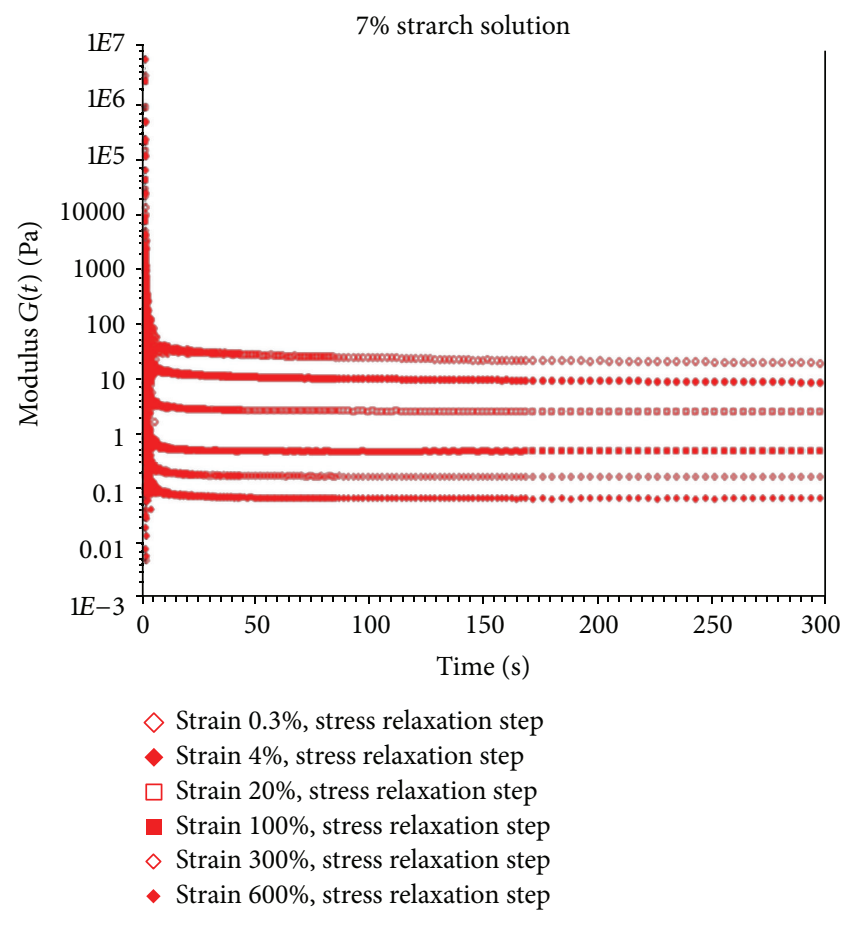

FIGURE 3: $G(t, \gamma)$ curve during the stress relaxation test under different strain for $0.3 \%, 4 \%, 20 \%, 100 \%, 300 \%, 600 \%$, respectively.

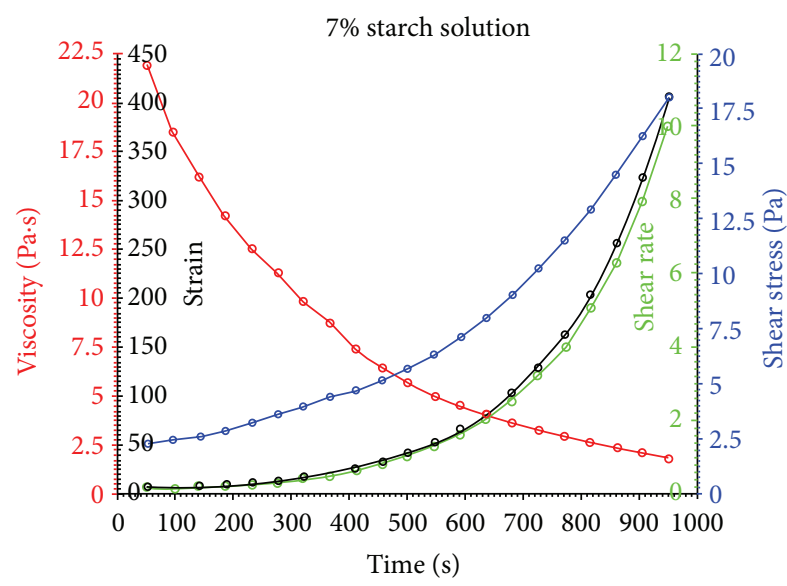

oooo Shear rate sweep from 0.1 to 10 (flow step)

Figure 4: Shear rate step flow test.

The verified experiment was carried out, and the result was shown in Figures 4 and 5.

In the shear rate sweep test, the time and shear rate are known. Therefore, the strain can be calculated by shear rate and time. The above parameters were taken into (14) and the predict, result was shown in Figure 5.

Viscosity-shear rate curve of starch solution was shown in Figure 6.

It indicates that the viscosity of starch solution will become thin with the shear rate increasing.

The predicted results indicate that the material function constructed with memory function describes the flow behavior during the shear rate step test. The shape of predicts 


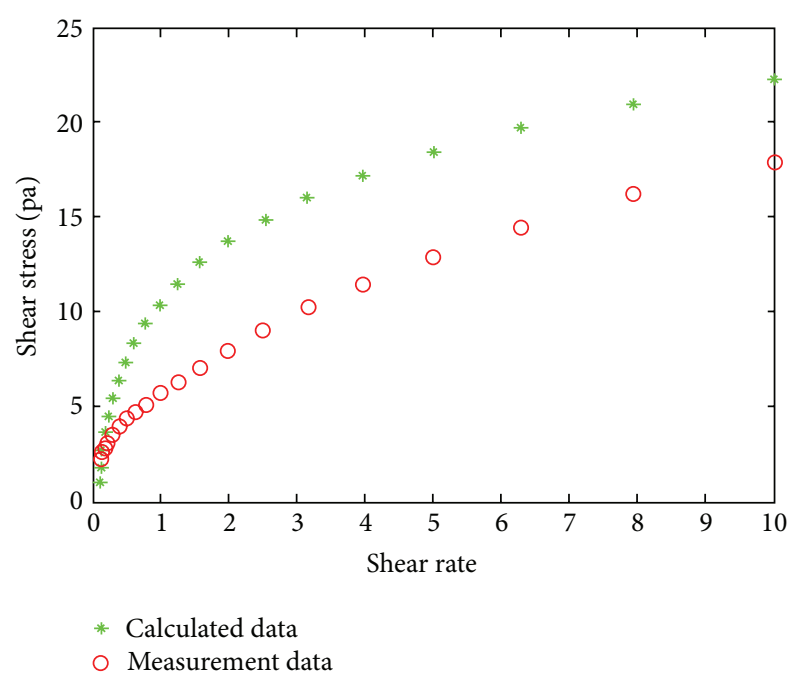

Figure 5: Predicted result of shear stress during the shear rate sweep test.

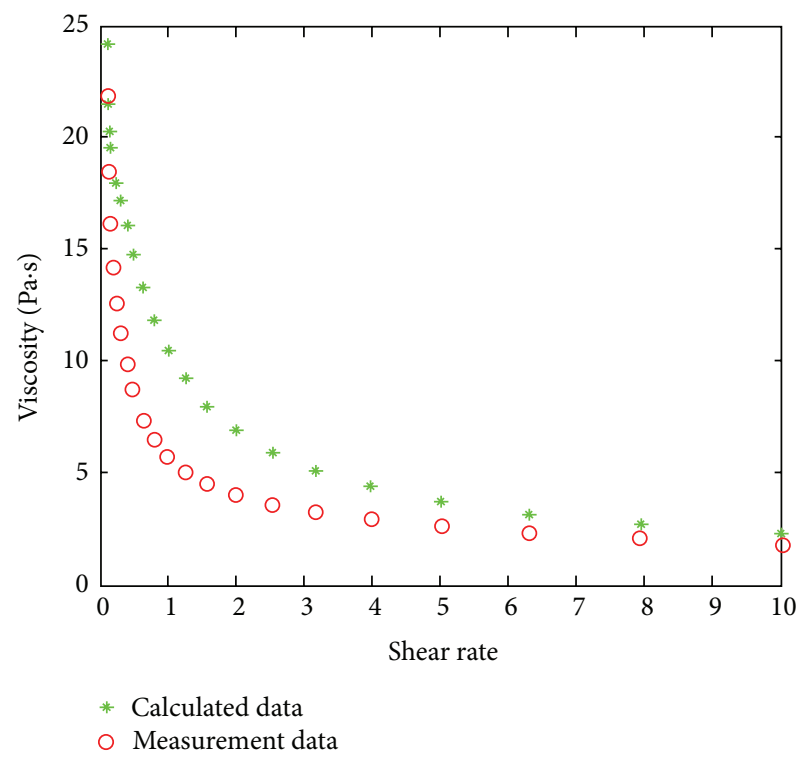

FIgURE 6: Viscosity-shear rate curve.

line based on the material function was very similar to the shape of measured data line. When the shear rate increases to infinite, both the predicted data and test data are close to the same value.

\section{Conclusion}

The memory function of starch solution can be constructed by the shear relaxation test. Based on the memory function, the material function of starch solution was established to predict the shear stress and viscosity during the steady shear flow. The predicted result shows that the material function can be used to describe the behavior of starch solution during the shear process.

\section{Acknowledgment}

This work was supported by the Fundamental Research Funds for the Central Universities under Grant no. JUSRP11203.

\section{References}

[1] D. Eidam, W. M. Kulicke, K. Kuhn, and R. Stute, "Formation of maize starch gels selectively regulated by the addition of hydrocolloids," Starch, vol. 47, pp. 378-384, 1995.

[2] L. Quintieri, A. Monteverde, and L. Caputo, "Changes in prolamin and high resistant starch composition during the production process of Boza, a traditional cereal-based beverage," European Food Research and Technology, vol. 235, no. 4, pp. 699709, 2012.

[3] L. Duo and G. Dan, "Implementing high weight measurement precision in package machines by using 8031 single chip microcomputer," Journal of Tianjin University of Commence, vol. 4, pp. 8-14, 1994.

[4] C. Luo, "The calculation of the solid conveying volumetric ratio flow rate of the food extruder," Packaging and Food Machinery, vol. 26, no. 2, pp. 30-32, 2008.

[5] J. F. Steffe, Rheological Methods in Food Process Engineering, Freeman Press, East Lansing, Mich, USA, 1996.

[6] A. S. Lodge, Elastic Liquids, Academic Press, London, UK, 1964.

[7] A. S. Lodge and J. Meissner, "Comparison of network theory predictions with stress/time data in shear and elongation for a low-density polyethylene melt," Rheologica Acta, vol. 12, no. 1, pp. 41-47, 1973.

[8] M. Sugimoto, Y. Masubuchi, J. Takimoto, and K. Koyama, "Melt rheology of polypropylene containing small amounts of high molecular weight chain. I. Shear flow," Journal of Polymer Science B, vol. 39, no. 21, pp. 2692-2704, 2001.

[9] M. H. Wagner, "Analysis of time-dependent non-linear stressgrowth data for shear and elongational flow of a low-density branched polyethylene melt," Rheologica Acta, vol. 15, no. 2, pp. 136-142, 1976.

[10] M. H. Wagner, "Prediction of primary normal stress difference from shear viscosity data using a single integral constitutive equation," Rheologica Acta, vol. 16, no. 1, pp. 43-50, 1977.

[11] J. H. Yu, P. H. S. Santos, and O. H. Campanella, "A study to characterize the mechanical behavior of semi-solid viscoelastic systems under compression chewing: case study of agar gel," Journal of Texture Studies, vol. 43, no. 6, pp. 459-467, 2012.

[12] J. D. Ferry, Viscoelastic Properties of Polymers, John Wiley \& Sons, New York, NY, USA, 1980.

[13] K. Osaki, "On the damping function of shear relaxation modulus for entangled polymers," Rheologica Acta, vol. 32, no. 5, pp. 429-437, 1993.

[14] Q. Zheng, W. Wang, Q. Yu, J. Yu, L. He, and H. Tan, "Nonlinear viscoelastic behavior of styrene-[ethylene-(ethylenepropylene)]- styrene block copolymer," Journal of Polymer Science B, vol. 44, no. 9, pp. 1309-1319, 2006.

[15] F. A. Morrison, Understanding Rheology, Oxford university press, New York, NY, USA, 2001.

[16] W. Wang, Z. Lu, Y. Cao, J. Chen, J. Wang, and Q. Zheng, "Investigation and prediction on the nonlinear viscoelastic behaviors of nylon1212 toughened with elastomer," Journal of Applied Polymer Science, vol. 123, no. 3, pp. 1283-1292, 2012. 
[17] H. Watanabe, T. Sato, K. Osaki et al., "Rheological images of poly(vinyl chloride) gels. 4 . Nonlinear behavior in a critical gel state," Macromolecules, vol. 31, no. 13, pp. 4198-4204, 1998.

[18] V. H. Rolón-Garrido and M. H. Wagner, "The damping function in rheology," Rheologica Acta, vol. 48, no. 3, pp. 245-284, 2009. 


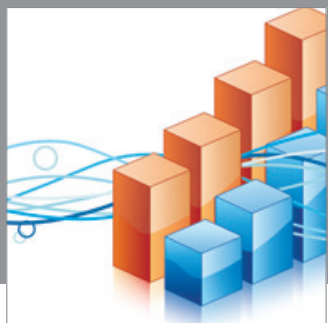

Advances in

Operations Research

mansans

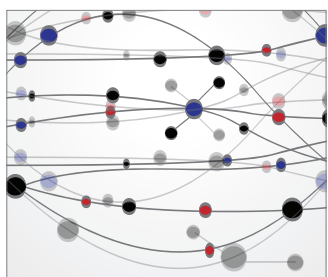

The Scientific World Journal
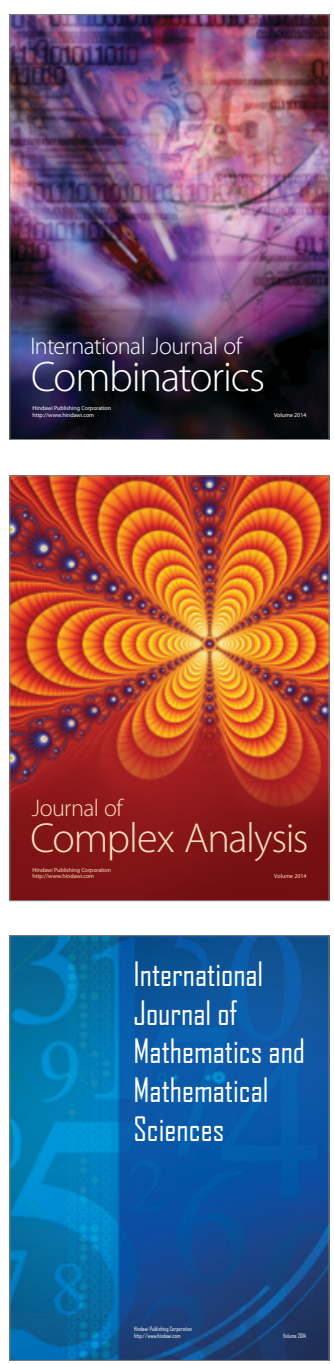
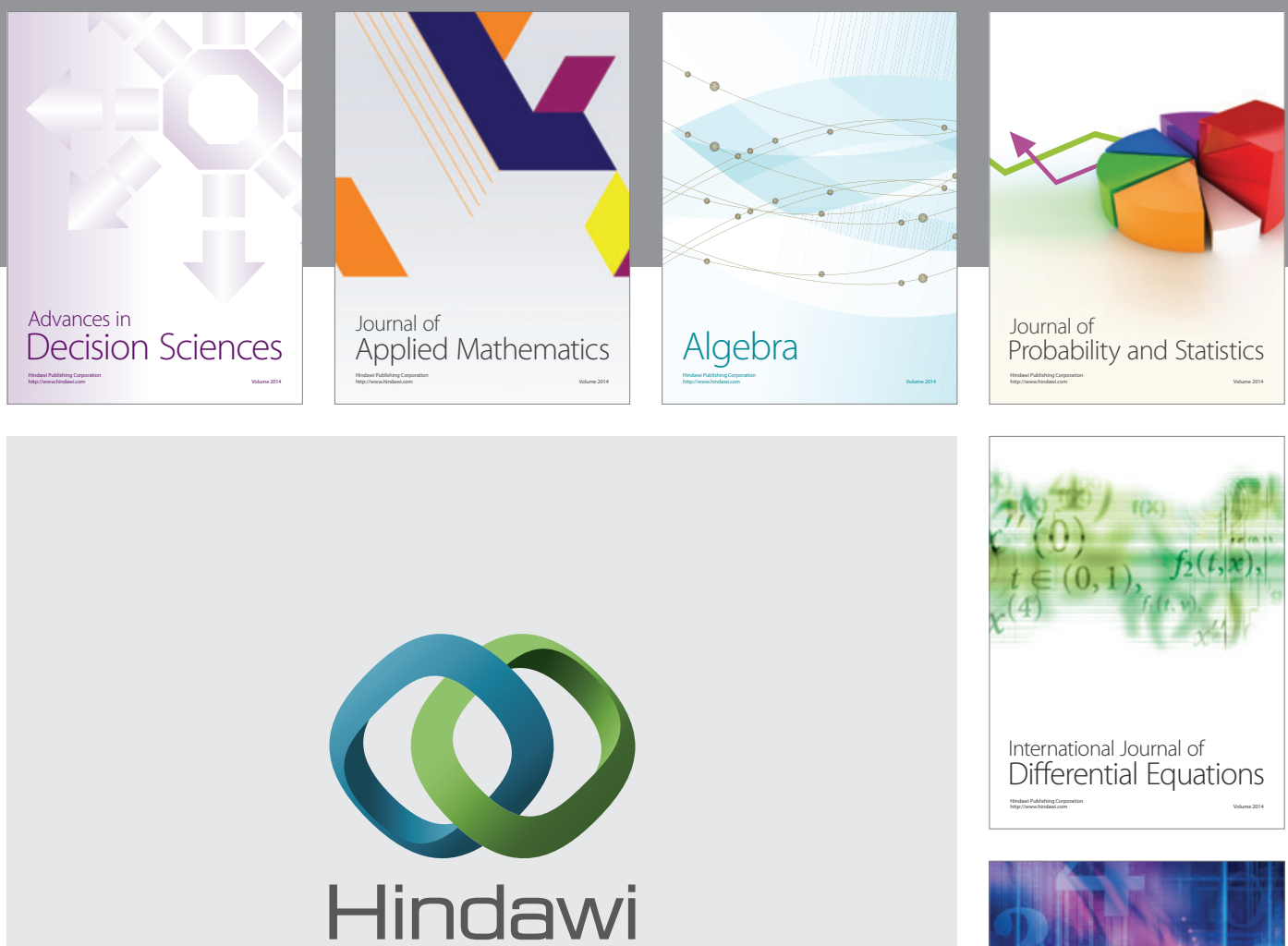

Submit your manuscripts at http://www.hindawi.com
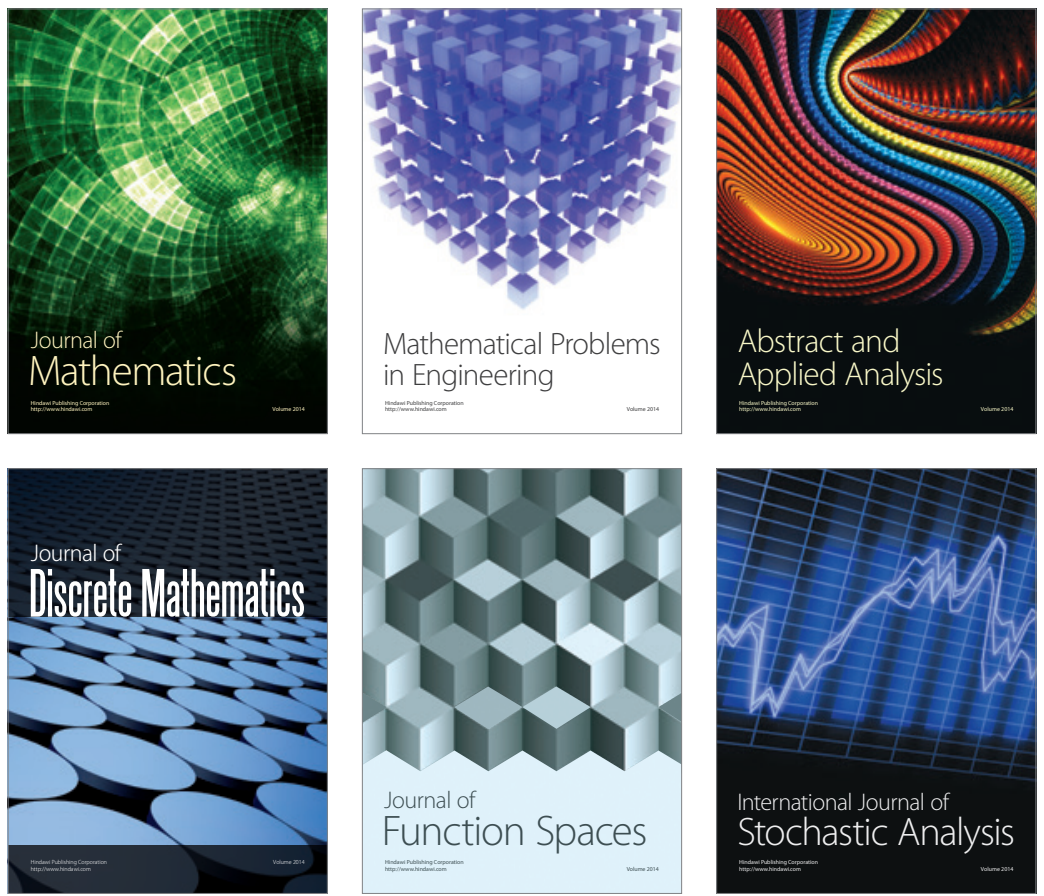

Journal of

Function Spaces

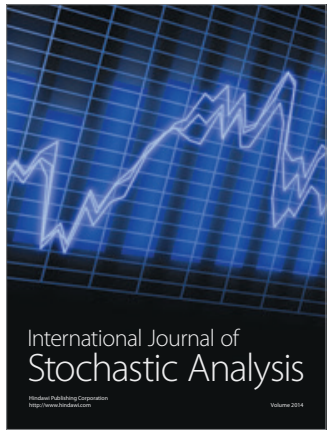

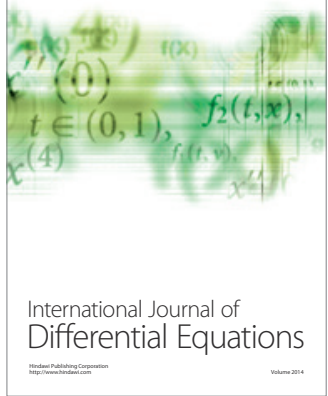
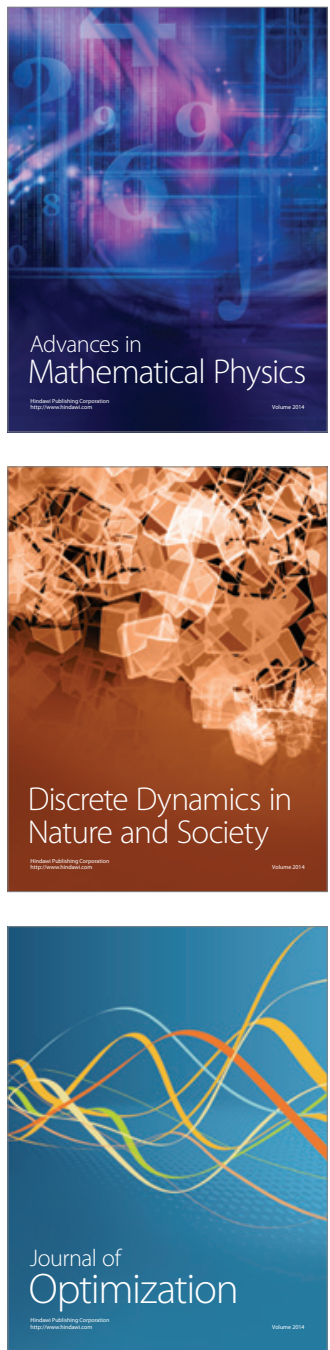